\title{
BMJ Open Health literacy in Italy: a cross-sectional study protocol to assess the health literacy level in a population-based sample, and to validate health literacy measures in the Italian language
}

Chiara Lorini, ${ }^{1}$ Francesca Santomauro, ${ }^{1}$ Maddalena Grazzini, ${ }^{2}$ Sarah Mantwill, ${ }^{3}$ Virginia Vettori, ${ }^{1}$ Vieri Lastrucci, ${ }^{1}$ Angela Bechini, ${ }^{1}$ Sara Boccalini, ${ }^{1}$ Alessandro Bussotti, ${ }^{4}$ Guglielmo Bonaccorsi ${ }^{1}$

To cite: Lorini C, Santomauro F, Grazzini M, et al. Health literacy in Italy: a cross-sectional study protocol to assess the health literacy level in a populationbased sample, and to validate health literacy measures in the Italian language. BMJ Open 2017;7:e017812. doi:10.1136/ bmjopen-2017-017812

- Prepublication history for this paper is available online. To view these files please visit the journal online (http://dx.doi. org/10.1136/bmjopen-2017017812).

Received 18 May 2017 Revised 1 September 2017 Accepted 14 September 2017

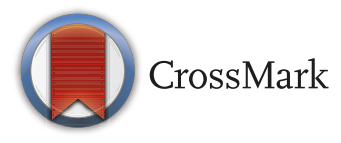

${ }^{1}$ Departement of Health Science, University of Florence, Florence, Italy

${ }^{2}$ School of Specialization in Hygiene and Preventive Medicine, University of Florence, Florence, Italy

${ }^{3}$ Department of Health Sciences \& Health Policy, University of Lucerne, Lucerne, Switzerland ${ }^{4}$ Careggi University Hospital, Florence, Italy

Correspondence to

Dr Chiara Lorini;

chiara.Iorini@unifi.it

\section{ABSTRACT}

Introduction Health literacy $(\mathrm{HL})$ concerns the knowledge and competences necessary for people to meet complex health demands. The aims of this study are to assess the level of $\mathrm{HL}$ in a sample using the Italian version of the Newest Vital Sign and the association of $\mathrm{HL}$ and selected antecedents with health outcomes, and to develop and validate the Italian version of the three Brief Health Literacy Screeners, two subjective numeracy items and the short form and the short-short form of the European Health Literacy Survey Questionnaire.

Methods and analysis The study adopts a crosssectional design and is being conducted in Florence, with information collected through telephone interviews. The population-based sample has been randomly selected using the registries of eight general practitioners (GPs). Based on a power calculation, 480 subjects will be included. Participants have been randomly offered two different questionnaires, each containing different $\mathrm{HL}$ measures. Data on sociodemographics and important antecedents and consequences of $\mathrm{HL}$ will be collected and the distribution of $\mathrm{HL}$ levels calculated. The mediating role of HL will be assessed using Preacher and Hayes' model. To assess the concurrent validity of the HL scales, correlation and receiver operating characteristic analyses will be performed.

Ethics and dissemination The study protocol has been approved by the Ethics Committee of the Area Vasta Centro. Results will be disseminated via scientific journals and conference presentations, and individual data made available to the GPs.

\section{BACKGROUND}

Health literacy (HL) concerns the knowledge and competences of individuals necessary for meeting the complex health demands of modern society. According to the conceptual framework of Sørensen et al 'HL is linked to literacy and entails people's knowledge, motivation and competences to access, understand, appraise and apply health information

\section{Strengths and limitations of this study}

- This is the first study in Italy to attempt to assess health literacy and its related antecedents and outcomes, and to validate a variety of different measures.

- The use of numerous measures will enable the systematic comparison of data with those obtained in other countries.

- The direct involvement of general practitioners (GPS) who signed the information sheet and agreed with the aims of the study could lead to high compliance.

- One limitation of this study is that the sample has not been randomly selected among people living in Florence, but from individuals registered as patients with different GPs.

- Another limitation is that the GPs have been recruited using convenience criteria, potentially introducing a selection bias.

in order to make judgements and take decisions in everyday life concerning healthcare, disease prevention and health promotion to maintain or improve quality of life during the life course'. In their framework, the authors identified 12 dimensions of HL, as well as proximal and distal factors (antecedents) that influence HL and related outcomes (consequences). Distal factors include societal and environmental factors, whereas proximal factors are situational and personal determinants such as general literacy, individual characteristics, and prior experience with illness and the healthcare system. On the other hand, the consequences at the individual and population levels refer to health service use and health costs, health behaviour and health outcomes, participation and empowerment in health issues, and equity and sustainability 
of public health issues. Despite this and other conceptual models (see, eg, the models of Nutbeam, ${ }^{2}$ PaascheOrlow and Wolf, ${ }^{3}$ and Schulz and Nakamoto ${ }^{4}$ ), to date the causal relationships between antecedents, HL and health outcomes are still unclear. Only a few studies have systematically investigated the role of $\mathrm{HL}$ as a mediator between antecedents and different health outcomes, while many authors have described HL as a cofactor or confounder in predicting health outcomes. ${ }^{5-13}$

Many tools exist to measure HL, but to date none of them are considered the gold standard. ${ }^{14} 15$ Measures largely vary in their approach and design, as well as in terms of their purpose. Some of them are screening tools that were originally developed for the clinical setting-to quickly identify patients with lower HL levels. Other tools were developed for the purpose of measuring the broader concept of HL to provide an in-depth assessment of the different dimensions of HL and exploring its relationship with determinants or outcomes. Moreover, HL measures are either performance based (objective) or self-reported (subjective). ${ }^{14-17}$ This means that they evaluate an individual's HL level by asking individuals to either directly apply their skills to a healthcare-related task or report on their skills, such as being confident enough to fill out medical forms on their own (see, eg, the studies of Chew et $a^{1819}$ ). Overall, there has been relatively little systematic research comparing the effectiveness of performance-based and self-reported measures, specifically with regard to possible relationships between HL and health outcomes. As suggested in a recent review, further studies that assess HL by using performance-based and self-reported tools at the same time are needed to clarify these aspects. ${ }^{20}$

In Italy, research on HL is still relatively new; only a few studies have assessed and published results on HL in Italian samples. ${ }^{21}{ }^{22}$ Therefore, the aims of this study are:

1. to assess the level of HL in a population-based sample in Florence, Italy, using the Italian version of the Newest Vital Sign (NVS-IT);
2. to assess the association of HL, selected antecedents and health outcomes, so as to evaluate whether HL is a mediator, a confounding factor or a cofactor in predicting the outcome variables;

3. to develop and validate the Italian version of three subjective Brief Health Literacy Screeners (BHLS) and two subjective numeracy items, and to assess their diagnostic accuracy in detecting subjects with limited HL in comparison to the NVS-IT (concurrent validation);

4. to develop and validate the Italian version of the short form (HLS-EU-Q16) and short-short form (HLS-EU-Q6) of the European Health Literacy Survey Questionnaire (HLS-EU), and to assess their diagnostic accuracy in detecting subjects with limited HL in comparison to the NVS-IT (concurrent validation).

\section{METHODS}

The study adopts a cross-sectional design and is being conducted in Florence, Italy and its surroundings. It started in February 2017 and will presumably end in November 2017, with information collected through telephonic interviews.

\section{Study population and sampling criteria}

The population-based sample was randomly selected from the registries of eight general practitioners (GPs) working in primary healthcare centres of the municipality of Florence-a sampling method that is also suggested by other authors (see, eg, the study of Toçi et $a t^{23}$ ) (figure 1). According to the regulations of the National Healthcare System and the Constitution of the Italian Republic, in Italy every citizen and foreign resident over the age of 18 has the right to be registered as a patient with a GP. Healthcare is provided by GPs free of charge to all patients. In the municipality of Florence, about 200

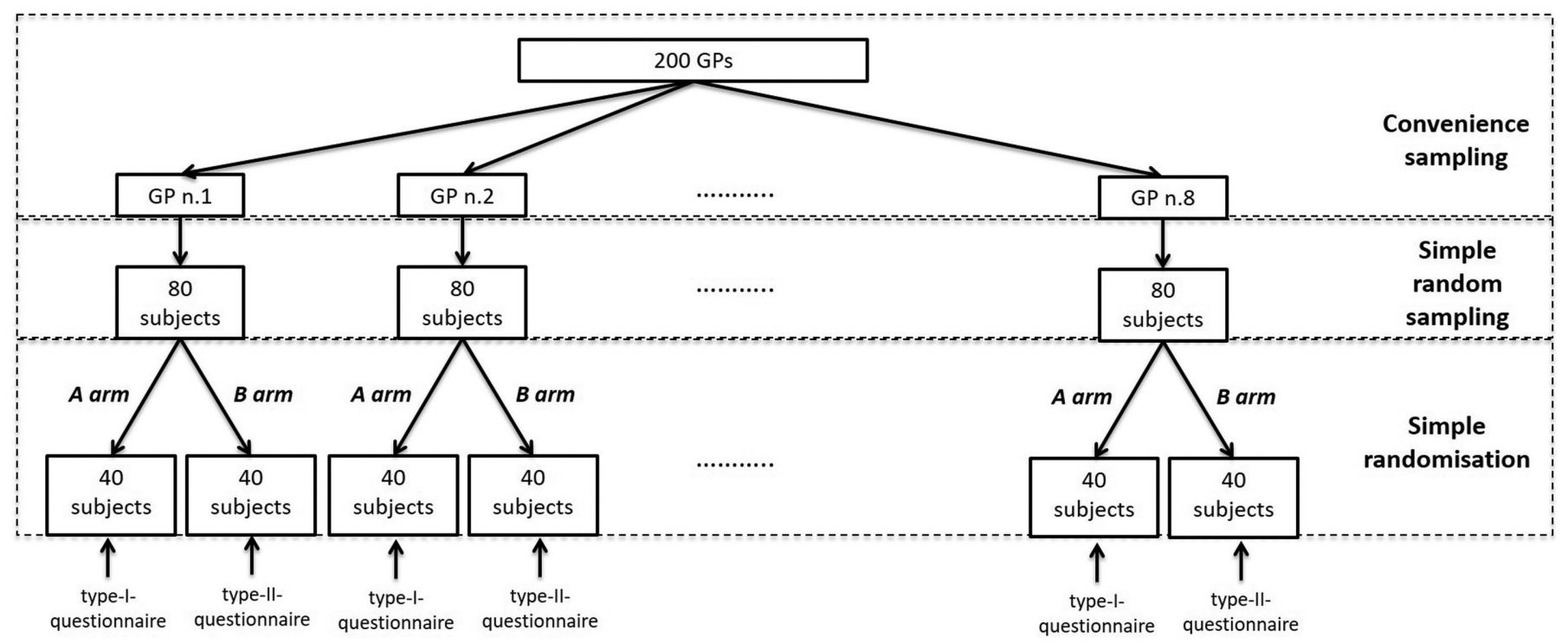

Figure 1 Sampling procedure. GP, general practitioner. 
GPs provide continuing and comprehensive healthcare to individuals who are registered as patients. For each GP, the number of registered and assisted patients is on average 1100, with a maximum limit of 1500 patients per doctor.

The GPs were recruited using convenience criteria. Both the President of the Provincial Medical Council and the representative of the GPs at the University Hospital of Florence informed their colleagues to join the study via either email or verbal contact. The first eight, to voluntarily join the study, were included. Each GP randomly selected 80 subjects among those registered as one of his/ her patients.

Inclusion criteria were the following: 18-69 years of age and Italian speaking (since the survey is conducted in Italian). Exclusion criteria included cognitive impairment, severe psychiatric diseases and end-stage diseases. Each GP verified the inclusion and exclusion criteria when selecting the sample.

Sample size was calculated based on the first aim of this study. Since no data on HL in Italy using Newest Vital Sign (NVS) as a measure is publicly currently available, data from the HLS-EU were used as a reference. Based on the original study that assessed HL across eight different European countries (Italy not included), ${ }^{6}$ Spain - with the highest rate of limited HL when assessed with the NVS-was chosen (34.3\%) as the expected value. Considering a CI of $95 \%$ and a margin of error equal to 0.05 , the sample size was established as 480 subjects. An oversampling of 160 individuals is planned to account for problems in recruitment (ie, subjects not available for telephonic interviews or refusal rates). If oversampling is not sufficient, a second random sample will be drawn.

Each subject is randomly allocated to one of the two arms of the study (A and B), according to the questionnaires used during the interview (type I and type II questionnaires, respectively).

\section{Procedures: data collection}

Each selected subject is contacted via postal mail. Subjects receive an information sheet signed by both the GP and the person in charge of the study, which includes a short description of the study, an invitation to participate and a consent form. Participants are asked to sign the consent form and return it via mail to the researchers in charge. The mail also contains the nutritional label of the NVS-IT. For further information, email addresses and telephone numbers of the researchers are provided in the letter. After receipt of the signed consent forms, the subjects are contacted over phone for the interview.

Nine interviewers who are part of the research group make the phone calls. Written instructions on how to conduct the interview are drawn up and shared within the research team so as to standardise the procedure and limit interviewer bias. Each subject is randomly assigned to one of the nine interviewers and contacted a maximum of six times before being considered unreachable.
Table 1 Summary of the content of the two types of questionnaires that will be used in the study

\begin{tabular}{lll}
\hline Questionnaire sections & $\begin{array}{l}\text { Questionnaire } \\
\text { type I }\end{array}$ & $\begin{array}{l}\text { Questionnaire } \\
\text { type II }\end{array}$ \\
\hline $\begin{array}{l}\text { General section } \\
\text { Health literacy measurements }\end{array}$ & $\mathrm{X}$ \\
NVS-IT & $\mathrm{X}$ & $\mathrm{X}$ \\
The three-item BHLS & $\mathrm{X}$ & \\
Two subjective numeracy & $\mathrm{X}$ & \\
items & & $\mathrm{X}$ \\
HLS-EU-Q16 and HLS- & & \\
EU-Q6 & & $\mathrm{X}$ \\
Health outcome variables & $\mathrm{X}$
\end{tabular}

BHLS, Brief Health Literacy Screener; HLS-EU-Q6, short-short form of the European Health Literacy Survey Questionnaire; HLS-EU-Q16, short form of the European Health Literacy Survey Questionnaire; NVS-IT, Italian version of the Newest Vital Sign.

Data are collected using an electronic database and anonymised by assigning a numeric code to each recruited person.

To meet the aims of the study, two questionnaires (type I and type II) have been drawn up, each containing the sections on the possible antecedents and consequences of HL, as well as different items to assess HL (table 1). Both questionnaires have a general section that includes questions on sociodemographics, familial data (antecedents) and health-related outcomes. In addition, both questionnaires include the NVS-IT items. However, whereas the type I questionnaire has three BHLS and two subjective numeracy items, the type I questionnaire contains a section with the HLS-EU-Q16.

The questionnaires have both been pretested with a small sample of volunteers, and take about 20-25 min, depending on the questionnaire.

\section{Measurements}

Newest Vital Sign

The NVS is a commonly used objective measure of HL. It was originally developed in the USA for English speakers and Spanish speakers. ${ }^{24}$ However, in recent years, the NVS has seen increased application in countries other than the USA, ${ }^{65-30}$ including Italy (NVS-IT). ${ }^{31}$ Moreover, it was originally developed to be administered by face-toface interviews, although in one study, a web-based survey was conducted $^{29}$ and in another it was self-administered. ${ }^{32}$ To the best or our knowledge, no published studies have reported NVS data collected through telephonic interviews.

The NVS-IT consists of an ice cream nutrition label, with seven associated questions that measure literacy and numeracy. It produces a final score ranging from 0 to 6 , allowing subjects to be classified in three categorieshigh likelihood of limited HL (score: $0-1$ ), possibility of limited HL (score: 2-3) and adequate HL (score: 4-6). This instrument takes very little time to be administered 
(3-5 min), and has been found to be easily applicable to people in different settings. ${ }^{33} 34$ Since the information needed to answer the questions has to be derived from the nutritional label, the label is included in the postal mail to be sent to the GP-assisted participants.

\section{HL and numeracy screeners}

In addition to the NVS-IT, the type I questionnaire also contains items of two other measurement tools-three BHLS and two subjective numeracy items. Both measures are self-reported with Likert-type responses, validated for predicting NVS scores by a previous study. ${ }^{35}$

The three BHLS were initially developed and validated in English. ${ }^{18}$ They assess literacy, interaction, comprehension and confidence (self-efficacy) skills. Even though they were originally developed for the clinical setting, they have seen increased application in population-based studies. ${ }^{36-41}$

The two subjective numeracy items-adapted from the STAT-confidence that assesses people's confidence in understanding medical statistics ${ }^{42}$ - were initially also validated in English. The two questions assess an individual's confidence in medical statistics by evaluating numeracy, comprehension, application/function, decision-making and confidence. Both tools produce a score to measure the HL levels.

\section{HLS-EU-Q16 and HLS-EU-Q6}

In addition to the NVS-IT, the type II questionnaire contains the HLS-EU-Q16 and the HLS-EU-Q6 which are respectively the short form and the short-short form of the 47-item European Health Literacy Survey Questionnaire (HLS-EU-Q47). The HLS-EU was developed in the aim of measuring and comparing HL levels of populations in different European countries. Based on the conceptual model proposed by Sørensen et al, ${ }^{1}$ a European Unionfunded working group developed the HLS-EU-Q47. ${ }^{43}$ It includes 47 items covering 12 subdomains (including domains such as accessing and obtaining, understanding or appraising information relevant to healthcare, disease prevention and health promotion). It is a self-reported tool with Likert-type responses ('very easy', 'fairly easy', 'fairly difficult', 'very difficult') and an associated final score that measures interaction, comprehension, information seeking, application/function, decision-making/ critical thinking, evaluation, responsibility, confidence and navigation skills. Based on the extended version, two short versions were developed by selecting 16 items for the HLS-EU-Q16 and 6 items for the HLS-EU-Q6. Even though the reduction in the number of items has decreased the informative value and theoretical scope of the measure, it has increased its utility by reducing its administration time, while maintaining strong correlations to the full instrument. ${ }^{44}$

To generate the score for the HLS-EU-Q16, answers are dichotomised ('don't know' answers are coded as missing values). 'Fairly difficult' and 'very difficult' are both coded with 0 (zero), whereas 'fairly easy' and 'very easy' are both coded with 1 . The HLS-EU-Q16 score is a sum score (range: 0-16) and according to the final score, three levels of HL have been defined: inadequate HL (0-8); problematic HL (9-12); sufficient HL (13-16).

Scores for the HLS-EU-Q6 are calculated similarly to those of the HLS-EU-Q47 (from 1 for 'very difficult', to 4 for 'very easy'). The final score is the mean score that is calculated if at least five of the six items are completed and range from 1 to 4 . According to the final score, three possible levels of HL have been defined: inadequate HL (1-2); problematic HL (2-3); sufficient HL (3-4).

\section{Translation of HL measurements}

The English versions of the BHLS, two subjective numeracy items, the HLS-EU-Q16 and the HLS-EU-Q6 have been translated and adapted using standard procedures, including forward and backward translation (performed by Italian and English native speakers). A final Italian version was drafted and then shared and discussed with members of the research group. ${ }^{45}$ Table 2 reports on the Italian versions of the tools.

\section{Antecedents}

Data on antecedents of HL were collected according to the proposal of the HLS-EU ${ }^{6}$ and the Italian lifestyle surveillance system PASSI (Progress by Local Health Units towards a Healthier Italy). ${ }^{46}$ In addition to including questions on gender, birth year, nationality, number of years living in Italy (for those who were born abroad), educational level and marital status, the questionnaire also has questions on the number of family members living in the same household, whether one has ever received training or is/has been employed in the field of healthcare, employment status (currently having a paid job), financial situation, and whether a family member or a friend normally accompanies him/her to medical appointments.

\section{Health outcome variables}

According to the Sørensen model ${ }^{1}$, many outcomes are associated with HL. In line with what has been already made public by the HLS-EU, ${ }^{6}$ the following information will be collected: self-assessed health status (excellent, very good, good, so-so/fair, bad), weight and height to calculate body mass index, health services used in the last 12 months (number of doctor visits, hospital admissions, emergency department visits and use of healthcare services such as dentists, physiotherapists, psychologists, dieticians or opticians. Responses are coded as follows: 0, 1-2 times, 3-5 times, 6 times or more, don't know/ refusal). To measure health status, subjects are asked about long-term illnesses (illnesses that have lasted or are expected to last for at least 6 months), coded in four categories: yes, more than one; yes, one; no; don't know/ refusal.

\section{Statistical analysis}

Statistical analysis will be performed using IBM SPSS Statistics for Windows, V.24.0 (IBM) and StataIC V.11 


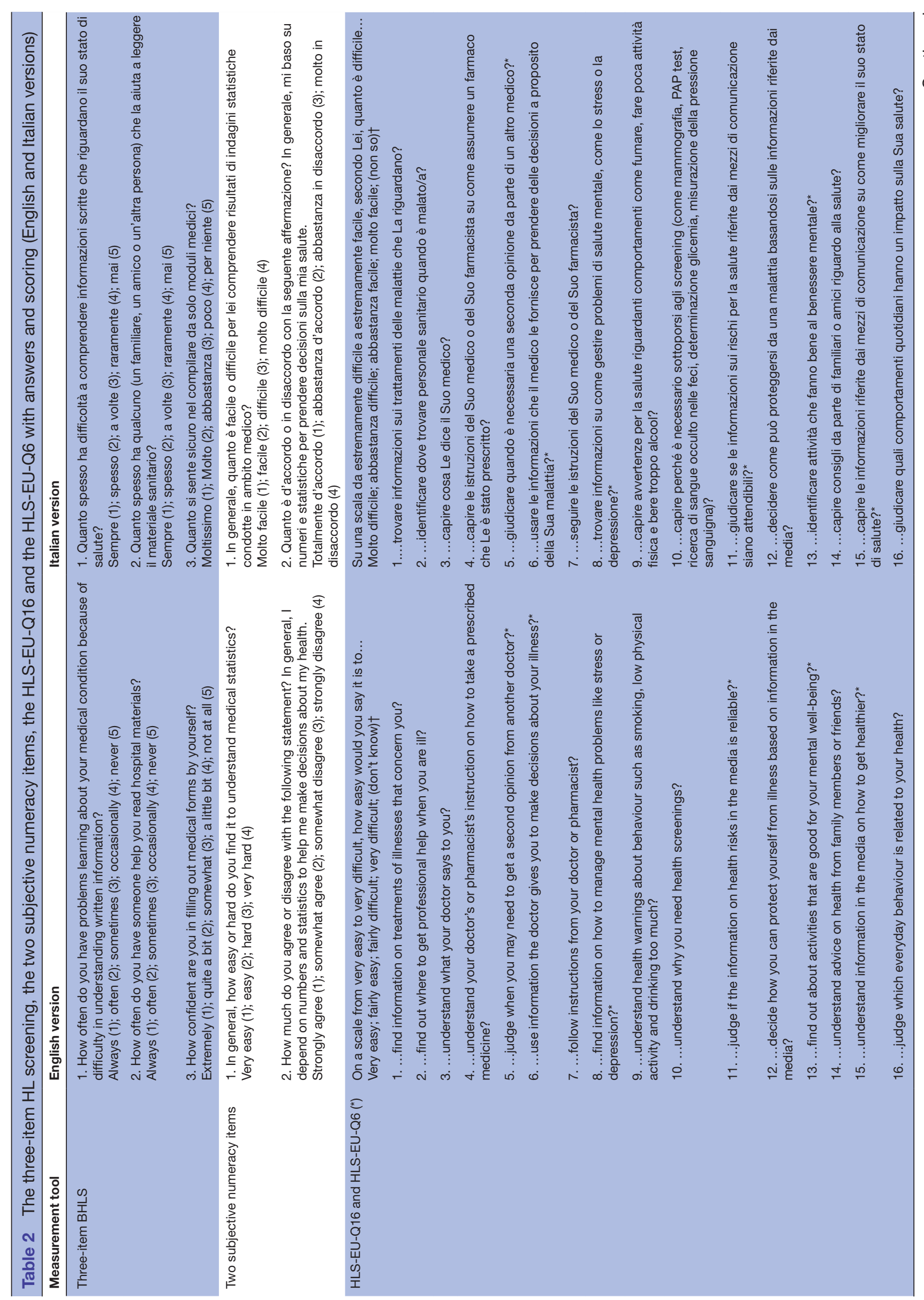


(StataCorp). For each analysis, an alpha level of 0.05 will be considered as significant.

Prior to the main analysis, data will be assessed for non-normality.

Descriptive statistics will be calculated and data will be presented as percentage or mean \pm SD. Associations between variables will be tested using Fisher's exact test for categorical data and the Student's t-test or analysis of variance for continuous data. Linear regression analysis will be performed to assess the linear relationships between HL and covariates. Receiver operating characteristic (ROC) analysis will be performed for validation. According to the aims of the study, different analyses will be carried out.

Aim 1. To assess the level of HL in a population-based sample in Florence, Italy, using the Italian version of the NVS-IT.

The distribution of HL levels will be calculated with the results projected back to the target population through inference analysis aimed at calculating estimates for the target population.

Aim 2. To assess the association of HL, selected antecedents and health outcomes, so as to evaluate whether $\mathrm{HL}$ is a mediator, a confounding factor or a cofactor in predicting the outcome variables.

Linear regression analyses will be performed to assess the linear relationship between NVS-IT scores, antecedents and health outcomes. In the first step, the association between antecedents (independent variables) and NVS-IT scores (dependent variable) will be assessed, followed by an assessment of the association between NVS-IT (independent variable) and health outcomes (dependent variable).

The potential mediating role of HL will be assessed using the Preacher and Hayes' model ${ }^{47}$ which is based on a normal theoretical approach and bootstrapping approach for obtaining reliable intervals between variables. ${ }^{48}{ }^{49}$ This approach supports the traditional methods of Baron and Kenny ${ }^{48}$ in studying the effects of mediating variables. ${ }^{50}$

Aim 3. To develop and validate the Italian version of three BHLS and two subjective numeracy items, and to assess their diagnostic accuracy in detecting subjects with limited HL compared with the NVS-IT (concurrent validation).

Aim 4. To develop and validate the Italian version of the short form (HLS-EU-Q16) and short-short form (HLS-EU-Q6) of the HLS-EU, and to assess their diagnostic accuracy in detecting subjects with limited HL, compared with the NVS-IT (concurrent validation).

To assess the concurrent validity (the degree of agreement between two different tools in measuring the same concept) and the diagnostic accuracy of the HL scales, correlation (Pearson or Spearman) and ROC analyses will be performed. In order to verify that the thresholds suggested in the original validation studies are capable of distinguishing individuals with lower HL levels from individuals with higher HL levels, the sensitivity, specificity, 
positive and negative predictive values and total accuracy will be calculated for each potential threshold value. Based on the already validated NVS-IT, two different analyses will be performed. The first analysis will consider participants with 'adequate HL' on the NVS-IT as being highly literate and those with 'possibility of limited HL' and 'high likelihood of limited HL' as being less literate. The second analysis will use a different grouping and assign those with 'Possibility of limited HL' on the NVS-IT to the highly literate group. The area under the ROC curve will be used as a measure of accuracy. ${ }^{51}$ To assess the level of agreement, Cohen's kappa will also be calculated.

Since the HLS-EU-Q16 has been developed from the HLS-EU-Q47 using Rash analysis, ${ }^{44}$ this analysis will be performed on the Italian version of the HLS-EU-Q16 to describe the 'test-item difficulty', its strengths and weaknesses. $^{52}$

\section{Ethics and dissemination}

The study has been designed following the principles of the Declaration of Helsinki. The protocol has been approved by the Ethics Committee of the 'Area Vasta Centro' (Local Health Unit of Central Tuscany, Careggi University Hospital and Meyer University Children's Hospital; Ref. CEAVC: 10113, 01 December 2016). As briefly described above, after data collection, information will be anonymised by assigning a numerical code to each participant. Any original identifiable information will be destroyed once the study has been completed.

Results will be disseminated through peer-reviewed scientific journals and conference presentations. Moreover, grouped data will be made available to GPs. Finally, the results will be discussed with policy-makers of the Tuscany Region.

\section{DISCUSSION}

\section{Strengths and limitation}

To the best of our knowledge, this is the first study in Italy that comprehensively attempts to assess HL and its related antecedents and outcomes and to validate a variety of different measures.

HL levels will be assessed in a population-based sample via use of a validated measure (NVS-IT), which has been applied in different countries and contexts ${ }^{25-30} 32$ and has also been used in the HLS-EU. ${ }^{6}$ This will allow for systematic comparison of data with those obtained in the eight countries involved in the European survey. Comparability will be made easier via use of a population-based sample of adults, similar to those included in the HLS-EU. In addition, given that the NVS-IT is already validated in Italian, the analysis will enable the systematic testing of the concurrent validity of the newly validated measures.

In this study, telephone interviews are used to collect NVS-IT data, while in almost all the studies face-to-face interviews are used. To date, no data have been published concerning the comparison of NVS data collected using different methods of administration. Compared with face-to-face interviews, telephone interviews offer several advantages, ${ }^{45}$ namely, the elimination of any bias caused by the appearance of the interviewer, lower costs related to the transfers of the interviewer or the person interviewed and lower administrative costs. Moreover, there is some evidence that people are more likely to report health-related events on the phone rather than in face-toface interviews. ${ }^{45}$ The potential problems with telephone interviewing are instead as follows: the respondent may seek help from another person at home, and there is no assurance who the person is at the other end on the line. This second risk is difficult to completely eliminate, while the first one is minor, ${ }^{45}$ since the interviewer would be able to notice the involvement of other people (ie, the person on the phone would have to repeat each question or use the hands-free mode).

Another strength concerns the HL measures themselves, as they have already been validated in different languages and/or settings. Therefore, the diagnostic accuracy of the newly validated measures will be compared with that identified in other studies. In any case, as noted by Reeve and Basalik, ${ }^{53} \mathrm{HL}$ tests were primarily designed to detect illiteracy, so they often show a ceiling effect when used in the general population. The ceiling effect can significantly skew distribution, giving rise to concern about attenuated correlation. In our study, this phenomenon might be observed for NVS-IT, BHLS and two subjective numeracy items, while the HLS-EU-Q47 and its short forms have initially been validated in the general population. Any possible ceiling effect or skewed distribution will be considered in the statistical analysis.

Another strength of this study is the direct involvement of the GPs who have signed the information sheet and agreed with the aims of the study. Due to their strong involvement, participants might be more motivated to participate in the study, which is expected to result in higher compliance (see, eg, the study of Toçi $e t a l^{23}$ ).

One limitation of this study is that while the sample was randomly selected among people living in Florence, it includes individuals registered as patients with different GPs. Moreover, the GPs were recruited using convenience criteria, which might introduce a selection bias. To curb some of the bias, GPs were chosen according to their geographical location, in the aim of covering different districts in Florence that may present different characteristics of the residents. Furthermore, the variable that identifies the subject's GP, as well as the number of registered patients, will be considered and included as a confounder in the analyses.

\section{Implications}

This study has a number of important implications. First of all, the results of the study will be useful in assessing the ability of community members to seek, locate and use health services. Moreover, obtaining validated HL measures in Italian will support current research efforts on HL and related outcomes in Italy-both at a population-based level and in the clinical setting. As suggested 
by other authors, ${ }^{44}$ it is proposed to use the HLS-EU-Q6 for research other than HL-specific research in Italy. More specifically, the current plan is to integrate the HLS-EU-Q6 as a covariate in the Italian lifestyle surveillance system PASSI.

In addition, since the results will be presented and discussed with the Regional policy-makers, including the General Directors of Local and Hospital Health Units, data on HL will also be taken into account when planning community-based health and marketing activities, educational strategies to be used in educational campaigns or campaigns to support the introduction of new services, screening initiatives (eg, bowel or skin cancer) and vaccination programmes. ${ }^{54}$

At a patient level, an important implication of the study relates to the direct involvement of the GPs. Apart from becoming more familiar with the concept of HL and its assessment tools, GPs will receive individual data for their patients, which will allow them to address findings in their day-to-day practice and potentially improve their care relationships.

Contributors CL, SM and GB: conception and design; drafted the article; gave final approval of the version to be submitted. FS, MG, W, VL and AIB: conception and design; gave final approval of the version to be submitted. AnB and SB: drafted the article; gave final approval of the version to be submitted.

Competing interests None declared.

Patient consent Obtained.

Ethics approval Ethics Committee of the 'Area Vasta Centro' (Local Health Unit of Tuscany-Center, Careggi University Hospital and Meyer University Hospital; Ref. CEAVC: 10113, 01 December 2016).

Provenance and peer review Not commissioned; externally peer reviewed.

Open Access This is an Open Access article distributed in accordance with the Creative Commons Attribution Non Commercial (CC BY-NC 4.0) license, which permits others to distribute, remix, adapt, build upon this work non-commercially, and license their derivative works on different terms, provided the original work is properly cited and the use is non-commercial. See: http://creativecommons.org/ licenses/by-nc/4.0/

(C) Article author(s) (or their employer(s) unless otherwise stated in the text of the article) 2017. All rights reserved. No commercial use is permitted unless otherwise expressly granted.

\section{REFERENCES}

1. Sørensen K, Van den Broucke S, Fullam J, et al. Health literacy and public health: a systematic review and integration of definitions and models. BMC Public Health 2012;12:80.

2. Nutbeam D. The evolving concept of health literacy. Soc Sci Med 2008;67:2072-8.

3. Paasche-Orlow MK, Wolf MS. The causal pathways linking health literacy to health outcomes. Am J Health Behav 2007;31(Suppl 1):19-26.

4. Schulz PJ, Nakamoto K. Health literacy and patient empowerment in health communication: the importance of separating conjoined twins. Patient Educ Couns 2013;90:4-11.

5. Berkman ND, Sheridan SL, Donahue KE, et al. Health literacy interventions and outcomes: an updated systematic review. Evid Rep Technol Assess 2011;199:1-941.

6. HLS-EU Consortium. 2012. Comparative report on health literacy in eight EU member states. The European health literacy survey HLS-EU. http://ec.europa.eu/chafea/documents/news/Comparative report_on_health_literacy_in_eight_EU_member_states.pdf

7. Osborn CY, Cavanaugh K, Wallston KA, et al. Health literacy explains racial disparities in diabetes medication adherence. $J$ Health Commun 2011;16(Suppl 3):268-78.
8. Schillinger D, Barton LR, Karter AJ, et al. Does literacy mediate the relationship between education and health outcomes? A study of a low-income population with diabetes. Public Health Rep 2006;121:245-54.

9. Hahn EA, Burns JL, Jacobs EA, et al. Health literacy and patientreported outcomes: a cross-sectional study of underserved Englishand Spanish-speaking patients with type 2 diabetes. $J$ Health Commun 2015;20(Suppl 2):4-15.

10. Meppelink CS, Smit EG, Diviani N, et al. Health literacy and online health information processing: unraveling the underlying mechanisms. J Health Commun 2016;21:109-20.

11. Gwynn KB, Winter MR, Cabral HJ, et al. Racial disparities in patient activation: evaluating the mediating role of health literacy with path analyses. Patient Educ Couns 2016;99:1033-7.

12. Bennett IM, Chen J, Soroui JS, et al. The contribution of health literacy to disparities in self-rated health status and preventive health behaviors in older adults. Ann Fam Med 2009;7:204-11.

13. Amit Aharon A, Nehama H, Rishpon S, et al. Parents with high levels of communicative and critical health literacy are less likely to vaccinate their children. Patient Educ Couns 2017;100:768-75.

14. McCormack L, Haun J, Sørensen K, et al. Recommendations for advancing health literacy measurement. $J$ Health Commun 2013;18(Suppl 1):9-14.

15. Haun JN, Valerio MA, McCormack LA, et al. Health literacy measurement: an inventory and descriptive summary of 51 instruments. J Health Commun 2014;19(Suppl 2):302-33.

16. Jordan JE, Osborne RH, Buchbinder R. Critical appraisal of health literacy indices revealed variable underlying constructs, narrow content and psychometric weaknesses. J Clin Epidemiol 2011;64:366-79.

17. Altin SV, Finke I, Kautz-Freimuth $S$, et al. The evolution of health literacy assessment tools: a systematic review. BMC Public Health 2014;14:1207.

18. Chew LD, Bradley KA, Boyko EJ. Brief questions to identify patients with inadequate health literacy. Fam Med 2004;36:588-94.

19. Chew LD, Griffin JM, Partin MR, et al. Validation of screening questions for limited health literacy in a large VA outpatient population. $J$ Gen Intern Med 2008;23:561-6.

20. Kiechle ES, Bailey SC, Hedlund LA, et al. Different measures, different outcomes? A systematic review of performance-based versus self-reported measures of health literacy and numeracy. $J$ Gen Intern Med 2015;30:1538-46.

21. Palumbo R, Annarumma $C$, Adinolfi $P$, et al. The Italian health literacy project: insights from the assessment of health literacy skills in Italy. Health Policy 2016;120:1087-94.

22. Palumbo R, Annarumma C, Adinolfi $P$, et al. The missing link to patient engagement in Italy. J Health Organ Manag 2016;30:1183-203.

23. Toçi E, Burazeri G, Myftiu S, et al. Health literacy in a populationbased sample of adult men and women in a South Eastern European Country. J Public Health 2016;38:6-13.

24. Weiss BD, Mays MZ, Martz W, et al. Quick assessment of literacy in primary care: the newest vital sign. Ann Fam Med 2005;3:514-22.

25. van Schaik TM, Jørstad HT, Twickler TB, et al. Cardiovascular disease risk and secondary prevention of cardiovascular disease among patients with low health literacy. Neth Heart $J$ 2017;25:446-54.

26. Hoffman S, Marsiglia FF, Nevarez L, et al. Health literacy among youth in Guatemala city. Soc Work Public Health 2017;32:30-7.

27. Protheroe J, Whittle R, Bartlam B, et al. Health literacy, associated lifestyle and demographic factors in adult population of an English city: a cross-sectional survey. Health Expect 2017;20:112-9.

28. Fransen MP, Leenaars KE, Rowlands G, et al. International application of health literacy measures: adaptation and validation of the newest vital sign in The Netherlands. Patient Educ Couns 2014;97:403-9.

29. Suka M, Odajima T, Okamoto M, et al. Reading comprehension of health checkup reports and health literacy in Japanese people. Environ Health Prev Med 2014;19:295-306.

30. Rowlands G, Khazaezadeh N, Oteng-Ntim E, et al. Development and validation of a measure of health literacy in the UK: the newest vital sign. BMC Public Health 2013;13:116.

31. Capecchi L, Guazzini A, Lorini $C$, et al. The first Italian validation of the most widespread health literacy assessment tool: the newest vital sign. Epidemiol Prev 2015;39(4 Suppl 1):124-8.

32. Couture ÉM, Chouinard MC, Fortin M, et al. The relationship between health literacy and quality of life among frequent users of health care services: a cross-sectional study. Health Qual Life Outcomes 2017;15:137. 
33. Bonaccorsi G, Santomauro F, Donzellini M, et al. Health literacy among paid caregivers of elderly people: preliminary results of a Tuscan study. Eur J Public Health 2016;26(Suppl 1):75.

34. Bonaccorsi G, Grazzini M, Pieri L, et al. Assessment of Health Literacy and validation of Single-Item Literacy Screener (SILS) in a sample of Italian people. Ann Ist Super Sanità 2017;53:205-12.

35. Stagliano V, Wallace LS. Brief health literacy screening items predict newest vital sign scores. J Am Board Fam Med 2013;26:558-65.

36. Moran MB, Chatterjee JS, Frank LB, et al. Individual, cultural and structural predictors of vaccine safety confidence and influenza vaccination among hispanic female subgroups. J Immigr Minor Health 2017:19:790-800.

37. Veldwijk J, van der Heide I, Rademakers J, et al. Preferences for vaccination: does health literacy make a difference? Med Decis Making 2015;35:948-58.

38. Kallenberg FG, IJspeert JE, Bossuyt PM, et al. Validation of an online questionnaire for identifying people at risk of familial and hereditary colorectal cancer. Fam Cancer 2015;14:401-10.

39. Smedberg J, Lupattelli A, Mårdby AC, et al. Characteristics of women who continue smoking during pregnancy: a cross-sectional study of pregnant women and new mothers in 15 European countries. BMC Pregnancy Childbirth 2014;14:213.

40. Vallance JK, Eurich DT, Gardiner PA, et al. Utility of telephone survey methods in population-based health studies of older adults: an example from the Alberta Older Adult Health Behavior (ALERT) study. BMC Public Health 2014;14:486.

41. Castañeda SF, Malcarne VL, Foster-Fishman PG, et al. Health care access and breast cancer screening among Latinas along the California-Mexican border. J Immigr Minor Health 2014;16:670-81.

42. Woloshin S, Schwartz LM, Welch HG. Patients and medical statistics J Gen Intern Med 2005;20:996-1000.

43. Sørensen K, Van den Broucke S, Pelikan JM, et al. Measuring health literacy in populations: illuminating the design and development process of the European Health Literacy Survey Questionnaire (HLSEU-Q). BMC Public Health 2013;13:948.

44. Pelikan JM, Röthlin F, Ganahl K, 2014. Measuring comprehensive health literacy in general populations:validation of instrument, indices and scales of the HLS-EU study. 6th Annual Health Literacy Research Conference. Bethesda, Maryland:Hyatt Regency. http://www.bumc bu.edu/healthliteracyconference/files/2014/06/Pelikan-et-al-HARC2014-fin.pdf

45. Streiner DL, Norman GR. Health measurement scales. Oxford: Oxford University Press, 2008.

46. II portale dell'epidemiologia per la sanità pubblica.http://www. epicentro.iss.it/passi/

47. Preacher KJ, Hayes AF. Asymptotic and resampling strategies for assessing and comparing indirect effects in multiple mediator models. Behav Res Methods 2008;40:879-91.

48. Baron RM, Kenny DA. The moderator-mediator variable distinction in social psychological research: conceptual, strategic, and statistical considerations. J Pers Soc Psychol 1986;51:1173-82.

49. Hayes AF. Beyond Baron and Kenny: statistical mediation analysis in the new millennium. Commun Monogr 2009;76:408-20.

50. Kline RB. Principles and practice of structural equation modeling. New York: Guilford, 2005.

51. Fawcett T. An introduction to ROC analysis. Pattern Recognit Lett 2006;27:861-74.

52. Boone WJ. Rasch analysis for instrument development: why, when, and how? CBE Life Sci Educ 2016;15:rm4.

53. Reeve CL, Basalik D. Is health literacy an example of construct proliferation? A conceptual and empirical evaluation of its redundancy with general cognitive ability. Intelligence 2014;44:93-102.

54. Batterham RW, Hawkins M, Collins PA, et al. Health literacy: applying current concepts to improve health services and reduce health inequalities. Public Health 2016;132:3-12. 